\title{
El impacto económico y las posturas de los actores principales en un ámbito universitario digitalizado
}

\section{Economic Implications and Stakeholder Reactions in a Digital University Environment}

\author{
Wolfram Laaser \\ FernUniversität. Hagen. Alemania. \\ wolframlaaser@googlemail.com
}

\begin{abstract}
Resumen
Actualmente predomina una sustancial inseguridad sobre el futuro del eLearning, y en particular sobre el impacto de la digitalización en el sector educativo. Muchos que al inicio se mostraron entusiastas ahora son más escépticos sobre el futuro desarrollo de la enseñanza mediada por tecnología, mientras que algunos mantuvieron su actitud positiva y siguen buscando formas de promover e implementar su uso en las universidades. Menos discutidas son las implicaciones económicas que la digitalización puede tener sobre las partes interesadas de las universidades y su toma de decisiones; mientras que, en el campo de la educación a distancia en línea, un enfoque descriptivo de los costos y beneficios ha predominado hasta ahora.

Partiendo de la crítica sobre el criterio metodológico empleado en algunos estudios, se discutirá el posible impacto de la digitalización en una futura universidad digital desde tres ángulos diferentes: una perspectiva histórica, una perspectiva económica, y las actitudes de los actores y sus características. En el pasado el análisis descriptivo de costos y beneficios de los cursos en línea era la forma predominante para analizar la eficiencia comparativa de diferentes sistemas de educación. Al contrario, queremos focalizar algunos puntos claves para incentivar un debate sobre la economía de recursos digitales y sus efectos sobre los respectivos actores principales (stakeholders). Además, trataremos perspectivas a largo plazo respecto a los efectos de la digitalización de contenidos educativos en un mundo global.

Concluyendo que la digitalización siguió un desarrollo continuo impulsado particularmente por las universidades de educación a distancia; y que fenómenos como los MOOC no son tan "disruptivos" como algunos pretenden. En su lugar, las políticas nacionales, la sostenibilidad económica y el impacto de la digitalización en las diferentes partes interesadas, determinarán la forma futura de la "Universidad Digital", en caso de que tal universidad existiera.

Palabras clave: Economía de cursos en línea, contenido digital, Educación a distancia, Digitalización, eLearning, Cursos en línea, MOOCs, Universidad digital, Globalización.
\end{abstract}

\footnotetext{
Abstract

At present a substantial insecurity prevails about the future of eLearning and particularly about the future impact of digitalization on the educational sector. Those, who have been enthusiastic at the beginning are now more esceptical about the future development of teaching with digital media, others maintain their positive attitude and look for ways to promote and implement their use in the university. Less discussed are the economic implications that digitization may have on the universities stakeholders and their decision making. In the field of online and distance education a descriptive approach of the costs and benefits has been so far predominant. We will raise instead some points to initiate a discussion about the economics of digital educational ressources and the possibile reaction and impact of teachers, students and institutions. We will point out also some long-term perspectives that digitalization of education might have on a global level.

Concluding we will argue that digitilization of educational content and the respective applications followed a continuous development pushed especially by universities of distance education and that phenomena such as MOOCs are not as "disruptive" as some claim. Instead,
} 
national policies, economic sustainability and the impact of digitization on different stakeholders will determine the future form of the "Digital University" in case such a university exists.

Keywords: Economics of online courses, digital content, Distance education, Digitalization, eLearning, Online courses, MOOCs, Digital University, Globalization.

\section{Introducción}

El problema de pronósticos reside en que el desarrollo tecnológico además de ser rápido, es sensible a influencias económicas y políticas. Por dichos motivos, la consistencia y legitimidad de tales intentos no resulta fácil de determinar. Además, la metodología, el cómo se generan los datos y los resultados publicados, rara vez se documenta en detalle. La mayoría de los estudios se basan en cuestionarios en línea rellenados por actores del campo de educación, también llamados "expertos" (Allen \& Seaman, 2013), (Hart, 2015, Jones, (2017); para una crítica de los muy conocidos Horizon Reports del New Media Consortium (NMC, 2017) vea Baggaley (2015) y Watters (2017).

Pero no iremos a agregar otro pronóstico más, ya hay suficientes números en el mercado. En vez de ello, discutiremos algunas experiencias y logros hasta hoy, que pueden ser relevantes para el desarrollo futuro de los medios digitales en la educación superior. Además, nos centraremos en sus implicaciones para una futura "universidad digital", suponiendo que algo así como una universidad digitalizada pueda existir.

Las instituciones de educación a distancia, fueron las primeras en aplicar la digitalización al desarrollo y distribución de contenidos educativos. Por ejemplo, la FernUniversität de Hagen en Alemania, comenzó a experimentar con procesamiento de texto y conferencias por computadora a principios de los años ochenta (Laaser, 2017). Posteriormente se desarrollaron simulaciones didácticas, cursos en línea y laboratorios virtuales. La digitalización permitió una integración muy cercana de los diferentes medios, impulsados sobre todo por el desarrollo de Internet. Los formatos digitales que iban surgiendo se fueron añadiendo e integrado en sistemas complejos de gestión del aprendizaje, proporcionando espacios web para la comunicación sincrónica o asincrónica.

El desarrollo de los medios de comunicación en la educación a distancia y las aplicaciones tomadas de la world wide web, obligaron a las universidades convencionales a mantenerse al día con el rápido desarrollo, y hacer un mayor uso de las nuevas instalaciones para la enseñanza y el aprendizaje. Sin embargo, no estaban muy interesadas en desarrollar nuevo contenido digital, porque eso podría haber provocado resistencia por parte de los docentes, que aún continuaban dictando conferencias de manera tradicional. Pero por otra parte, les agradaba la idea de invertir en un mejor soporte de tecnológico, que los ayude en el trabajo administrativo.

Con respecto al sistema de gestión del aprendizaje, resultó que estos sistemas inicialmente se aislaron de las bases de datos empleadas con fines administrativos. Pero tiempo después, la combinación de sistemas de gestión del aprendizaje y los datos de los procesos administrativos tales como matriculación, certificación, programación de conferencias, y costos, condujo la evolución hacia los sistemas de gestión del campus, que están en uso hoy en día. Este desarrollo sigue en curso y tiene un gran potencial para el desarrollo futuro (Shacklock, 2016) (Kaltura (2018).

Desde finales de los años noventa, la producción y distribución de contenidos didácticos pudo facilitarse a un bajo costo, a través de sistemas de gestión del campus, instalaciones de conferencias y redes de servicios. Por lo tanto, las universidades convencionales

El impacto económico y las posturas de los actores principales en un ámbito universitario digitalizado. Wolfram Laaser

Página 2 de 20 
pudieron ajustar su modelo de enseñanza, para integrar el aprendizaje fuera del campus y las actividades de enseñanza, a sus programas. El resultado, un sistema mixto denominado "Blended Learning", que incluye grabaciones de conferencias en línea, auto aprendizaje a través de medios electrónicos, trabajo grupal sincrónico y asincrónico, y actividades presenciales. En la actualidad, la combinación de estos componentes del sistema, varían entre universidades e incluso entre facultades o departamentos. El término "Blended Learning", es un ejemplo de una palabra de moda que en efecto no excluye ningún modo de enseñanza específico. Podemos afirmar que la innovación denominada "educación a distancia", en cierta medida, se esparció por todo el sector educativo. Debido a su éxito curiosamente perderá su significado como modalidad de enseñanza particular.

\section{La economía de la "Universidad Digital"}

\section{El legado de la Educación a Distancia}

Las universidades de enseñanza a distancia han aplicado desde sus primeros años, un patrón industrializado de desarrollo, producción, y distribución, que incluye equipos de elaboración de cursos, unidades de enseñanza modular, gestión y organización profesional. La era digital cambió la estructura de costes, tanto para las instituciones a distancia como para las presenciales; pasando de la inversión en edificaciones, a la inversión en contenido, informática, y formación del personal. Los materiales educativos impresos perdieron su liderazgo como medio central para la enseñanza a distancia; y los formatoss digitales más económico o gratuito, posibilitaron un modo descentralizado de producción y distribución de contenidos digitales. La tecnología de comunicación cambió de, "uno a muchos", a una "comunidad en red"; con varios conglomerados que incluyen también a las redes sociales externas a la universidad.

Respecto a los costos, Wagner (1972) demostró que el gasto por estudiante en la British Open University, la famosa universidad de enseñanza a distancia, era aproximadamente un tercio de lo que invertían otras universidades británicas convencionales.

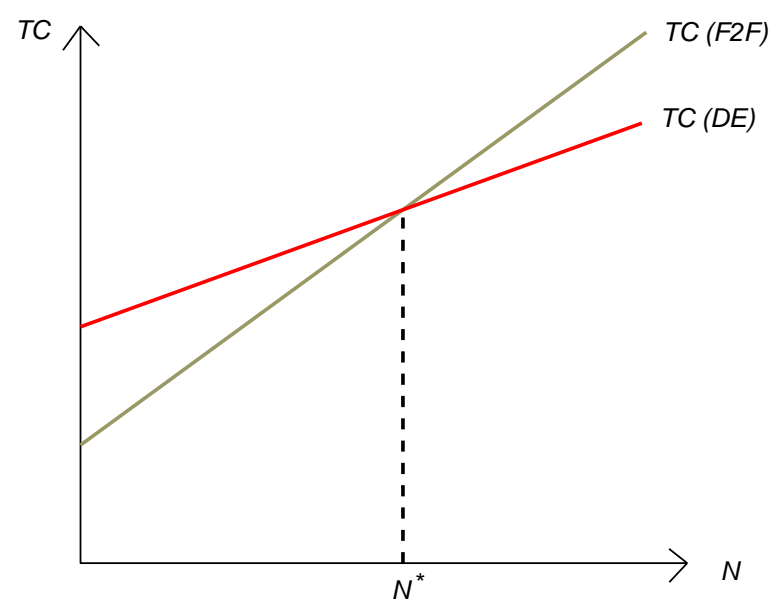

Figura 1: Costo de F2F y Educación a Distancia (Según Wagner, 1972).

$\mathrm{TC}=$ Costos Totales $; \mathrm{F} 2 \mathrm{~F}=$ presencial $; \mathrm{DE}=$ Educación a Distancia; $\mathrm{N}=$ Número de estudiantes.

El impacto económico y las posturas de los actores principales en un ámbito universitario digitalizado. Wolfram Laaser

Página 3 de 20 
A partir del diagrama, se puede observar que existe un nivel de matrícula que torna a la educación a distancia más barata que la educación presencial. Pero resulta discutible si el aprendizaje a distancia se puede delinear como se muestra en el diagrama, porque la calidad de la enseñanza y otros factores, también podrían ser relevantes para la comparación (e.g. Laaser (2008) Yenbamrung 1994).

\section{Blended Learning}

Este tipo de enfoque no es completamente válido para los escenarios actuales de blended learning. La introducción de segmentos de autoaprendizaje, facilitados por la grabación de conferencias u otro material digital (modelo de aula invertida), requiere la formación de profesores, la adquisición y actualización de software, y probablemente servicios permanentes de apoyo técnico para profesores y estudiantes. Estos costes pueden compensarse con una reducción de la inversión en la construcción de aulas y otros activos locales, pero incluso las salas de conferencias deben estar especialmente equipadas con buenos equipos de grabación y edición.

Uno de los problemas del blended learning como mezcla de clases presenciales con fases de aprendizaje autónomo mediado, es el ritmo del progreso individual de cada alumno, considerando experiencias de aprendizaje muy heterogéneas. Esto es particularmente válido para los Cursos Masivos en Línea (Massive Open Online Courses: MOOC), cuando se ofrecen sin restricciones de acceso y sin costo alguno. La coordinación, especialmente con respecto a las reuniones presenciales, es difícil y requiere mucho tiempo de preparación, tanto para los profesores como para los estudiantes. Este problema también fue publicado en un blog de Edsurge (Shah, 2017) donde se propone volver al viejo sistema de ofrecer los MOOCs con datos fijos de comienzo y fin.

Por otro lado el encuentro de estudiantes y profesores en vivo tiene alto valor emocional y social. Sin embargo, un sistema que se concentra en gran medida en la enseñanza mediada y la colaboración a través de redes, sin encuentros presenciales, pero con apoyo y retroalimentación por conferencias o foros en línea, puede ser todavía una alternativa económicamente atractiva. Probablemente la combinación de fases presenciales y mediadas (Blended Learning), no podrá lograr una reducción de costos comparable a la lograda por los sistemas de educación enteramente a distancia.

El impacto económico y las posturas de los actores principales en un ámbito universitario digitalizado. Wolfram Laaser 


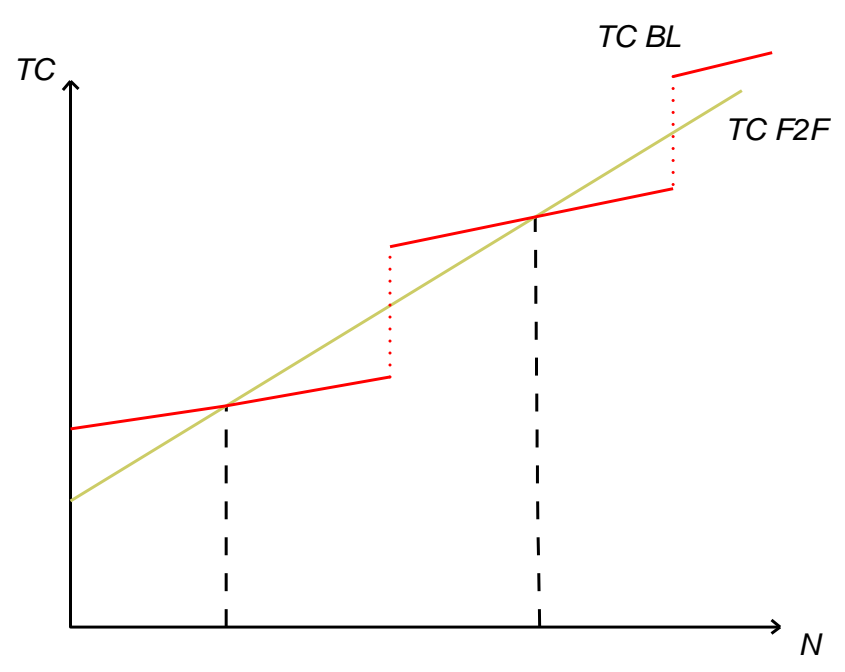

Fig.2: Costo total de un escenario combinado

Debido a las fases de auto aprendizaje, un aumento en el número de estudiantes requerirá menos horas de clases adicionales que en un modelo de enseñanza completamente presencial; de manera que parte de la carga de trabajo se transfiere a los estudiantes. Sin embargo, un salto en los costos fijos se producirá de vez en cuando, debido al costo adicional de las salas de conferencias, instalaciones de grabación y edición; necesarias para abastecer la mayor demanda. Además, todavía hay algunos costos variables, como el costo operacional de la plataforma, o las actualizaciones de software; existiendo un compromiso entre escalabilidad, y calidad educativa. Todo tipo de comunicación simultánea, ya sea electrónica o presencial, dificulta la explotación plena de las economías de escala debido al tiempo que duran las conferencias; que es caro, y tiene horarios rígidos para tales reuniones (Laaser, 2008).

\section{La "evolución disruptiva" de los MOOC}

Para profundizar e ilustrar los argumentos anteriores, veremos el debate actual sobre los MOOC, los cuales parecían ser una alternativa de bajo costo a las clases tradicionales, y al mismo tiempo representan una estrategia para entrar en los mercados mundiales. La matrícula masiva de estudiantes en los primeros "xMOOCs" (MOOCs del tipo ofrecido por las principales universidades estadounidenses), no fue apoyada por tutores o docentes. Los estudiantes recibieron conferencias en video, transmitidas o descargables, y algunos exámenes de opción múltiple. Inicialmente, el desarrollo y funcionamiento de los MOOCs fue financiado por grandes donaciones en los Estados Unidos, para que los programas pudieran ofrecerse gratuitamente. Pero luego algunos recortes de fondos, las universidades tuvieron que buscar otras fuentes de financiamiento.

Se pueden cobrar los certificados y los exámenes, ofrecer tutorías adicionales, o usar datos de estudiantes para enviar publicidad a fin de atraerlos a los cursos que exigen matricularse. Sin embargo, con el aumento de la matrícula y la creciente heterogeneidad de los participantes, la necesidad de ofrecer servicios de apoyo más específicos, como los tutoriales en línea o presenciales, aumentarán y extenderán la carga financiera de la

El impacto económico y las posturas de los actores principales en un ámbito universitario digitalizado. Wolfram Laaser

Página 5 de 20 
universidad. Y en caso de no proporcionar dicho apoyo, el MOOC terminará con altas tasas de deserción.

Por lo tanto, el concepto de XMOOC se encuentra ante un dilema: o baja la calidad del servicio acrecentando la deserción, o mejora la calidad aumentando el apoyo; financiado el gasto adicional con cuotas especiales para cubrir los costos de las plataformas, exámenes y certificaciones. En este último caso, el número de estudiantes será más limitado, y se hace obvio que el xMOOC finalmente resultará ser una oferta simplemente regular; sólo que no exige una cualificación previa para inscribirse en alguno de sus cursos.

Udacity y Coursera, dos de las grandes plataformas MOOC, ya han abandonado su política de ofrecer contenido digital, certificados o tutoriales de forma totalmente gratuita. Según Young (2018) Udacity ya considera los MOOCS como Muertos. Como expresa en un informe uno de los primeros promotores del MOOC: "La gran cantidad de estudiantes, llevó a encendidos reclamos para interrumpir la educación, y dejó un sector al borde del cambio sistémico. Sin embargo, desde la perspectiva de 2015, estas afirmaciones aparecen cada vez más erróneas, ya que los MOOC han demostrado ser simplemente una oportunidad adicional, en lugar de un desafío directo a la educación superior en sí "(Siemens, Gaševic \& Dawson, 2015, p.6).

Aunque puede resultar fascinante pensar en videoconferencias de clases sin costo para el participante, que puedan ser vistas por un número casi ilimitado de estudiantes en cualquier lugar y en cualquier momento, los MOOC representan básicamente una estrategia de marketing, mediante la cual parte del contenido de la enseñanza de una universidad se ofrece básicamente gratuito. El costo marginal para distribuir una copia adicional de una conferencia tiende a ser cero para la institución y las viejas economías de escala parecen reaparecer sólo hasta cierto punto.Pero las instituciones muy pronto buscan modelos de negocio para cubrir los gastos sustanciales (véase también Laaser, 2014.

\section{Digitalización y estructura de mercado}

Desde una perspectiva microeconómica, una universidad que basa su enseñanza principalmente en medios digitales, muestra algunas similitudes con la economía de la industria del software.

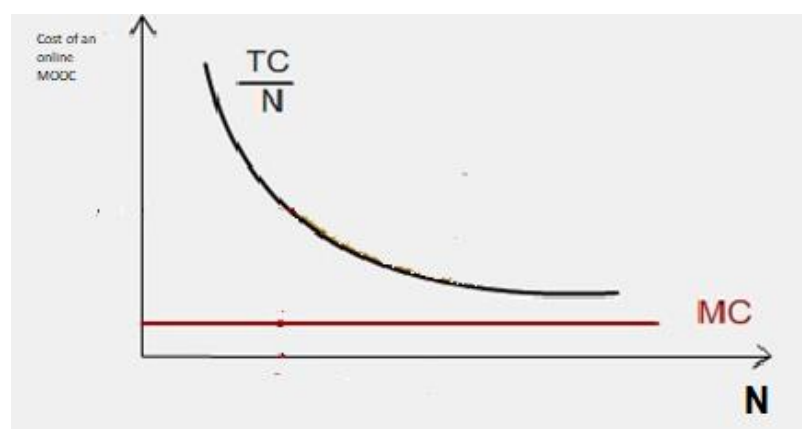

Figura 3: Costo total promedio y costo marginal del software (basado en Shy (2001))

La figura 3 representa una situación en la que el costo de producción por unidad (por ejemplo estudiantes matriculados), está dominado principalmente por el coste fijo de la

El impacto económico y las posturas de los actores principales en un ámbito universitario digitalizado. Wolfram Laaser

Página 6 de 20 
inversión inicial; y se caracteriza por unos costes de distribución muy bajos (MC). Mientras que el costo total por estudiante, disminuye a medida que aumenta el número de participantes $\mathrm{N}$.

El caso de la disminución del costo total promedio con el aumento de estudiantes, también ha sido una de las razones del rápido crecimiento de la enseñanza universitaria a distancia. ¿Habrá un efecto parecido con los XMOOCs en las universidades?

Se supone que el costo marginal de matricular a un estudiante adicional, permanece inicialmente constante en un nivel mínimo. Si elegimos un nivel de inscripciones de N1, entonces existe un precio correspondiente P1 que equilibra el costo y el beneficio. Cualquier resultado mayor que N1 genera beneficios netos, y la producción por debajo de N1 genera pérdidas. Este escenario tiende a favorecer la estructura de un mercado que representa un monopolio en la oferta, y se denomina "monopolio natural".

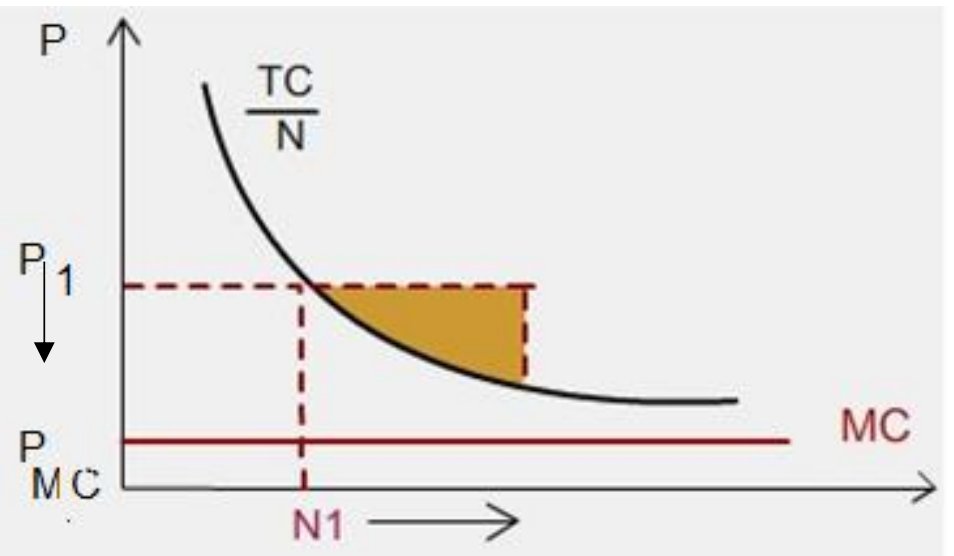

Fig. 4: Costo total promedio y costo marginal de software (basado en Shy, 2001)

En caso que las estructuras del costo de productos educativos digitalizados (como por ejemplo una maestría en línea ), correspondan a las características de las figuras 3 y 4 en un mercado privado competitivo, la competencia entre los proveedores bajará el precio hasta el nivel del precio $\mathrm{P}_{\mathrm{mc}}$, correspondiente al costo marginal.

A corto plazo, el costo fijo de la inversión inicial ya realizada se considerará como irrecuperable o "sunk cost" (costo no relevante para la política de precios a corto plazo debido a que ya se han realizado las respectivas inversiones). Por lo tanto, la competencia de precios en el mercado de contenidos educativos digitales relativamente similares, reduciría el precio hacia el costo marginal. Entonces, la competencia conduciría a un proceso de autodestrucción en el que la producción generaría pérdidas individuales. En cambio, si un monopolista proporciona toda la oferta del mercado, dispone del costo total promedio más bajo por alumno inscrito, y puede fijar el precio para maximizar en ese momento sus beneficios.

Las estructuras de mercado que resultan en monopolios naturales, son a menudo reguladas por los gobiernos para evitar el uso indebido de la posición dominante. Por tal motivo, pueden considerar al MOOC como un bien público y subsidiar hasta cierto punto su producción, para que el curso pueda ser ofrecido a los estudiantes de manera gratuita.

El impacto económico y las posturas de los actores principales en un ámbito universitario digitalizado. Wolfram Laaser 
Suponiendo que las subvenciones se limitan a la inscripción N1, servirían para cubrir principalmente el coste de desarrollo del curso, que puede considerarse como costo fijo del MOOC. E incluye algún costo marginal también, como por ejemplo, pago por uso de una plataforma que no es propia de la universidad.

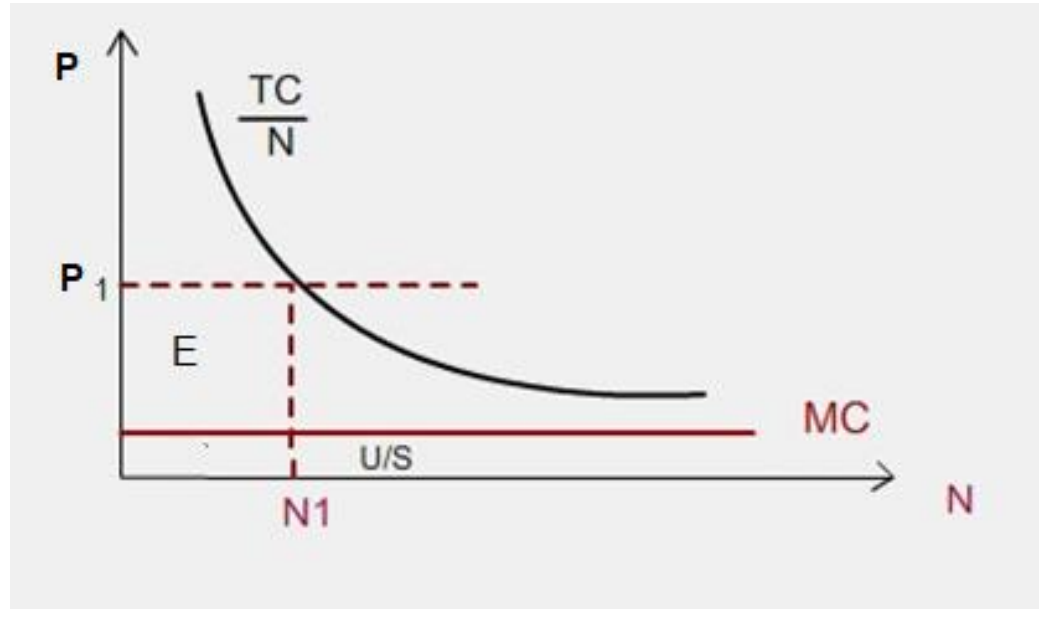

Figura.5 Financiamiento de MOOCs. Externo (E) universitario (U) estudiantes (S)

Si la universidad quiere extender la matrícula más allá de N1, entonces puede bajar el costo promedio total por alumno, pero no recibirá más financiamiento externo. A corto plazo sólo tiene que financiar el costo recurrente marginal adicional, pero se deben hacer algunas previsiones para cubrir luego, el costo de desarrollo de nuevos cursos.

Si la universidad está autorizada a cobrar un precio de acuerdo con el costo promedio total, el beneficio neto sería entonces cero; tal como lo requieren instituciones públicas sin fines de lucro, como las universidades públicas. Si el MOOC se ofrece de forma gratuita, dichos costos deben ser cubiertos por la universidad, y la demanda para la inscripción a los cursos no se detendrá antes de que se encuentre completamente satisfecha.

Sin embargo, con una matrícula adicional masiva, la calidad de los servicios podría deteriorarse debido a la imposibilidad de ofrecer un adecuado acceso a tutoriales y apoyo técnico. Quizás también se incremente la varianza en los niveles de los alumnos ingresantes, habilidades de lenguaje, patrones culturales, etc. Sin extender los servicios de apoyo, la calidad del curso disminuirá, así como también la motivación de los estudiantes para pagar los honorarios adicionales (utilidad marginal).

El impacto económico y las posturas de los actores principales en un ámbito universitario digitalizado. Wolfram Laaser 


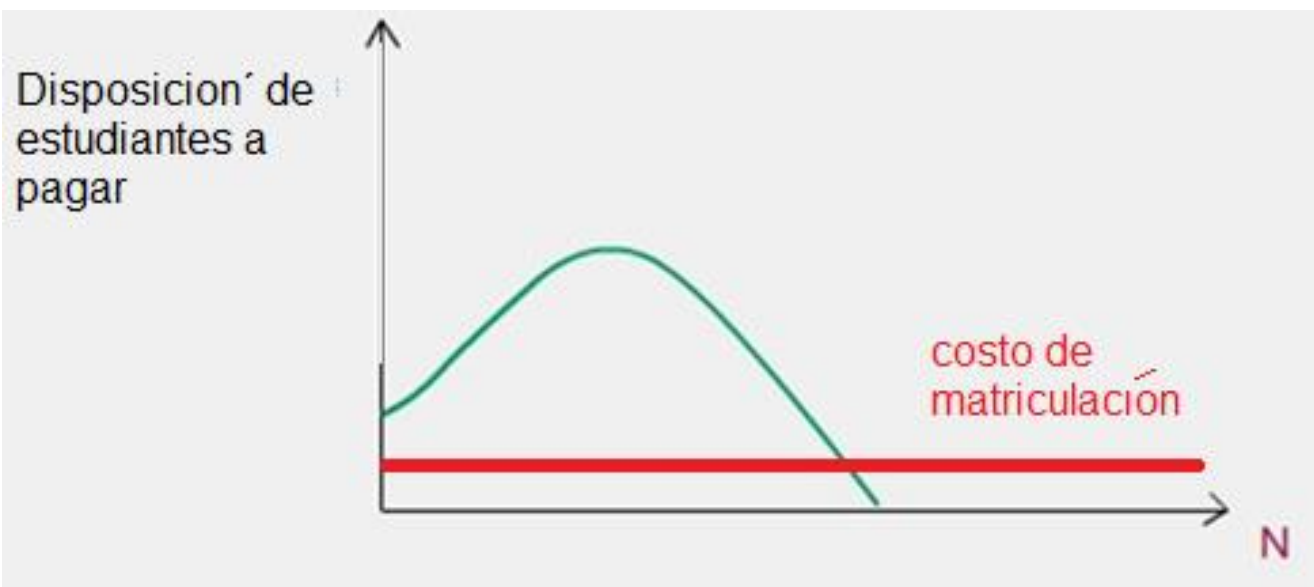

Figura 6. La disposición de los estudiantes a pagar honorarios por un MOOC

Si el estado financia completamente el costo total promedio, lo que significa que la inscripción es gratuita, las inscripciones no son infinitas; los estudiantes en este caso hacen su decisión en base de las esperadas ventajas o desventajas de la masividad del curso. Otro factor limitante son sus costos alternativos (oportunity costs) al no poder estudiar otro curso regular.

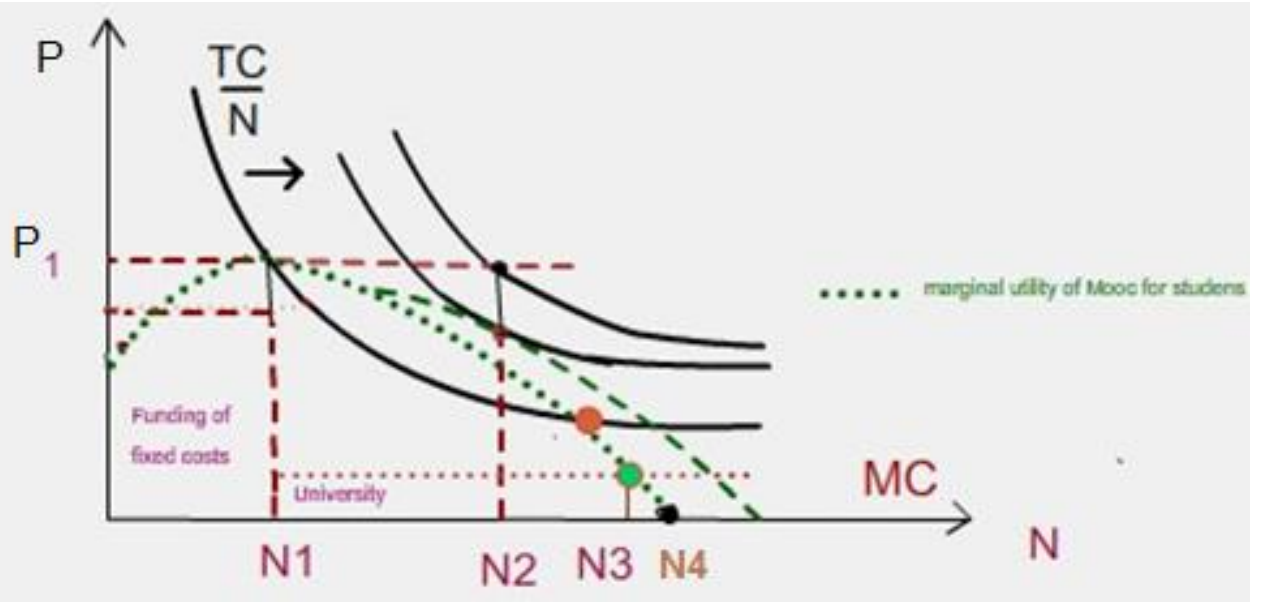

Figura 7: Impacto de altos números de participantes en cursos digitales en linea

En la figura 7 el número máximo de $\mathrm{N}$ para un curso gratuito es $\mathrm{N} 4$ suponiendo un nivel de apoyo actual ofrecido por la universidad. Cuando la universidad desea que los estudiantes paguen el costo marginal operativo en vez de la universidad misma, el número de inscritos no supera N3. En caso de que la universidad esté interesada de recuperar el nivel N4, debe gastar más dinero para los costos recurrentes y/o costo para equipos; lo que incrementa el nivel de gastos totales por estudiante, y la curva del costo promedio se traslada a la derecha. En qué medida la universidad logra un incremento en las inscripciones, depende de la voluntad de los estudiantes a contribuir o financiar esta

El impacto económico y las posturas de los actores principales en un ámbito universitario digitalizado. Wolfram Laaser

Página 9 de 20 
mejora de los servicios; y la institución no va a ser capaz de cubrir el costo total cuando termine el apoyo externo. Tampoco los estudiantes van a estar dispuestos de pagar más que una matricula mínima.

En países como los EE.UU las cuotas para los colegios ya son muy elevados, y será difícil pedir más. A corto plazo la universidad debe cubrir solamente el costo marginal adicional más allá de N1, y hasta N3 los estudiantes podrían pagar la oferta. Sin embargo, algunas previsiones presupuestarias siempre deben ser hechas para futuros cursos que no tengan financiamiento. Y se alcanzaría la situación socialmente ideal si el precio de la oferta de los Moocs corresponde al costo operativo o costo marginal.

Si la universidad desea mantener el nivel de calidad inicial, la curva del costo total promedio se desplazará hacia la derecha, debido a los gastos adicionales necesarios para mejorar los servicios. Estos podrían ser costos fijos adicionales, como actualizaciones de software, contenido, o la incorporación de tutoría por web casting, que también aumentaría el costo marginal. Por lo tanto, la curva de costo total promedio se desplazaría hacia la derecha. Y probablemente los gastos adicionales para el apoyo estudiantil modificarían también la curva de utilidad marginal; pero si la mejora de la calidad de la enseñanza es mayor que la disminución debido a una mayor matrícula, entonces la matrícula general puede ser aumentada.

Concluyendo, podemos decir que la universidad tiene diferentes opciones para fijar los precios de un MOOC. Los posibles precios oscilan entre cero y las tarifas que cubren el costo marginal, hasta el precio de acuerdo al costo total promedio relacionado con el número de inscriptos. Sin embargo, las universidades probablemente no serán capaces de cubrir todos los costos variables. Tratarán de ahorrar, ya sea reduciendo la calidad, automatizando los procesos administrativos, o agregando tarifas especiales más altas para mantener o ampliar el nivel inicial de calidad, mejorando los tutoriales u otros servicios actuales. Por lo tanto, la presión de financiar el MOOC mediante honorarios se incrementa con las inscripciones masivas, pero como institución sin fines de lucro, la universidad también tendría que respetar los precios a mediano plazo; y como los honorarios ya son muy altos, al menos en los Estados Unidos, sería difícil de lograr un aumento significativo. Como se demostró, el lado financiero puede tener efectos positivos o negativos en la inscripción y, consecuentemente en la calidad. Si al mismo tiempo se aumentan los honorarios y se reduce la financiación pública, no será posible financiar los costos recurrentes con el ingreso adicional generado por los pagos de los estudiantes. Ya que su contribución potencial se utiliza para sustituir los subsidios públicos, o servir para otros reclamos internos sobre el presupuesto de la universidad.

Aunque hemos explicado algunos argumentos sobre una cierta tendencia a actuar como monopolio, hay factores que limitan el poder de mercado, mientras que otros lo están fortaleciendo. Cualquier recién llegado tiene que invertir en el desarrollo tecnológico antes de ofrecer su producto, mientras que las empresas ya existentes pueden ofrecer, al menos temporalmente, su producto a coste marginal para expulsar a los recién llegados del mercado. Los nuevos participantes en el mercado van a elegir para sus productos campos bien específicos y confiar en el efecto de "bola de nieve" (snowball effect), lo que significa que un software es más atractivo cuanto más clientes lo usan (por ejemplo whatsapp, moodle, o facebook).

A medida que disminuye el coste de producción del contenido de la enseñanza en línea, (por ejemplo la grabación y edición de vídeo ya se puede hacer con un teléfono y subirla a

El impacto económico y las posturas de los actores principales en un ámbito universitario digitalizado. Wolfram Laaser

Página 10 de 20 
un espacio gratuito en la web), cualquier monopolio de software educativo u oligopolio debe tener en cuenta la entrada de nuevos competidores, ya que el costo de entrada al mercado es bajo. Y como estrategia para defender su fuerte posición en el mercado, las nuevas "start-ups" suelen ser simplemente adquiridas por las empresas que ya están en el mercado. Además, las empresas dominantes hacen un esfuerzo enorme para defender o ampliar su cuota de mercado, realizando grandes gastos en comercialización mediante el análisis de datos, para dirigirse de manera precisa al cliente potencial. Esto tiende a hacer que las empresas más pequeñas sean mucho menos visibles para el cliente.

Una tendencia análoga, de alta concentración de mercado, se puede observar en las publicaciones académicas digitales; donde prevalece una estructura de costos similar. Según Larivière, Haustein y Mongeon (2015) desde el advenimiento de la era digital de mediados de 1990, los principales editores aumentaron su participación en las producciones científicas publicadas. En 2013, alrededor del 70\% de los trabajos publicados en ciencias sociales, fueron de los cinco principales editores.

En otro sentido, varias universidades de los Estados Unidos utilizan una plataforma común para la distribución de sus xMOOC como Udacity o Coursera para reducir los costos de desarrollo y operación de los MOOCs (para "economías de alcance" ver Li \& Chen, 2012). Sin embargo, ya es visible que al menos en Europa no habrá una "plataforma europea MOOC" común, y probablemente ni siquiera una sola plataforma nacional, como han anunciado en Noruega; ya que el desarrollo de dicha plataforma puede hasta ser creado en Moodle, y adaptarse mejor a las necesidades de grupos institucionales más pequeños y homogéneos (Creelman, 2014), (Cooch, Foster, \& Costello, 2015).

Sin embargo, los presupuestos públicos están siendo presionados por el aumento de los presupuestos de educación, ya que se espera que se equilibren los salarios públicos con los del sector privado; problema que también es relevante para las universidades públicas. La consecuencia será que las actividades con altos costos como la tutoría, serán reemplazadas en gran medida por inteligencia artificial. Sustituir el tiempo de enseñanza y la evaluación, por la colaboración y la co-evaluación de los mismos estudiantes, no es una solución convincente y eficiente. Los alumnos necesitan organizar un aprendizaje significativo para ellos mismos, lo que podría alargar el tiempo de estudio y así llevar a un período de graduación más extenso; costoso para la economía de un país. Además, a la luz de experiencias pasadas respecto a la aplicación de la inteligencia artificial, ¿hasta qué punto el diálogo humano puede ser sustituido en la enseñanza superior por las máquinas? (véase en este contexto Endlich \& Sohoi, 2017). La educación superior es un segmento del sector educativo, donde los procesos repetitivos o los diálogos son menos frecuentes, y donde la mirada, el tono de la voz, la expresión corporal y demás, son importantes para establecer una buena relación.

A primera vista, parecería razonable estandarizar las MOOC y crear un mercado educativo más transparente que permita la acreditación compartida entre las distintas instituciones. De ser así, las negociaciones podrían entonces ayudar a las universidades a completar su plan de estudios, agregando materiales especiales que quizás serían demasiado caros para que una institución produjera por sí misma. En los Estados Unidos hasta ahora no hay normalización visible, ni existe un sistema común de puntos de crédito. El sistema educativo americano de educación superior se caracteriza en gran medida por universidades y fundaciones privadas, por lo tanto, los primeros MOOCs gratuitos que se ofrecían a las universidades más prestigiosas, tenían que crear sus propios ingresos después de la

El impacto económico y las posturas de los actores principales en un ámbito universitario digitalizado. Wolfram Laaser

Página 11 de 20 
financiación inicial; con ingresos propios mediante la certificación, o incluso por la venta de datos de los estudiantes a otras instituciones o cobrando por el apoyo tutorial.

En contraste con Estados Unidos, Europa avanzó en la normalización y acreditación de carreras con el sistema de clasificación ECTS (European Credit Transfer System). Sin embargo, al igual que en el pasado, el problema es que la cooperación entre las universidades sigue siendo muy deficiente a través de las fronteras nacionales, debido a las diferencias sociales, culturales y legales existentes. Por lo tanto, el mercado aún no está suficientemente desarrollado, y una normalización temprana o una regulación pueden llegar a obstaculizar las innovaciones.

Visionarios como Jeremy Rifkin (Rifkin, 2016) afirman que el bajo costo de los medios educativos, distribuidos casi al costo marginal, desafiarán al existente monopolio del conocimiento que la universidad tradicional todavía representa. La universidad (pública) es un proveedor de servicios que será reemplazado cada vez más por el acceso individual al contenido de la enseñanza, a costo cero, que también se puede crear, compartir y evaluar en colaboración entre pares.

La esperanza, de Rifkin y otros de que la economía de mercado capitalista se transforme en una sociedad en la que dominan los bienes colectivos y el intercambio de bienes y servicios auto-producidos, parece poco realista; tanto desde el punto de vista de la dinámica de una economía de mercado, como respecto a la distribución real del poder económico. Parece más bien que el sector educativo público se volvería más dependiente de la política de las grandes empresas internacionales.

¿Cuáles son los efectos más específicos de la digitalización, las redes y las TI en las universidades de enseñanza en línea? Discutiremos esto diferenciando el impacto en los actores interesados. Aspectos externos como los Ministerios de Educación e Industria, e internos como los líderes centrales (Rector, CEO, etc.), el personal administrativo, profesores y estudiantes.

\section{El impacto de la digitalización en los diferentes actores Los estudiantes}

Se espera que los estudiantes se conviertan en alfabetos digitales, capaces de identificar y utilizar los recursos de la web; que aprendan a crear contenido, comunicarse y cooperar a través de la web. Deben poder comprobar la corrección y credibilidad de la información web, y hacer un uso creativo de las nuevas opciones para el aprendizaje ubicuo. Si estos objetivos ya se han conseguido durante la educación escolar, podríamos obtener un alumno mucho más autónomo; que sea capaz de adquirir las competencias destacadas por la Comunidad Europea (véase, por ejemplo, Williams, Kear \& Rosewell, 2012).

Sin embargo, ¿cuál será el "lado oscuro" de esta modificación en la ecología social? Disminuirá la cohesión social y la interacción de los estudiantes con su entorno social directo, y serán en parte sustituidas por las identidades virtuales de los compañeros; que quizás puedan desviarse de su identidad real. La presentación en las redes sociales puede ser un signo de una cultura narcisista, y los estudiantes simpatizarán o no con personas que conocen sólo por su identidad virtual. Esto abre un gran potencial para la seducción y distribución de información o ideologías ocultas o simuladas (World Economic Forum, 2016).

Además, los estudiantes se distraerán más por la lectura de la pantalla; ya que la información que están acostumbrados a recibir, es diversa en el diseño, las fuentes y el

El impacto económico y las posturas de los actores principales en un ámbito universitario digitalizado. Wolfram Laaser

Página 12 de 20 
contexto. En consecuencia, pueden experimentar algunas dificultades para concentrarse en un problema, durante un período más largo de tiempo. Brabazon describe el problema: "A medida que progresa cada semestre, una mayor proporción de mis estudiantes están leyendo menos, haciendo menos referencias y escribiendo con menos audacia. Siempre habrá un $25 \%$ de la clase que son académicos rigurosos y comprometidos que pueden trabajar en modelos de aprendizaje centrado en el estudiante. Mientras que más del cincuenta por ciento, requieren una mayor orientación, atención y compromiso del personal docente para aprobar un curso... Estos problemas no son causados por Google. Pero si está facilitando la pereza, y el pensamiento complaciente "(Brabazon, 2014, capítulo 1, p.1).

Morgan cita a Hassan, respecto al incremento del espacio denominado "tiempo de red". "Cuanto más vivimos en la red (en un PC en el trabajo o en casa, en un PDA en el tren, o en la calle con un teléfono móvil), más habitamos su dominio temporalmente acelerado, con su potencial de desorientación y ritmo frenético "(Hassan, 2004, p.28). Según Hassan, el tiempo de la red conduce a la distracción crónica. Los estudiantes se enfrentarán a un entorno académico donde los objetivos sociales y la erudición académica, se interrelacionan con un creciente grado de influencias comerciales, ya sea en la escuela, en casa o en el lugar de trabajo; especialmente si miramos las redes sociales.

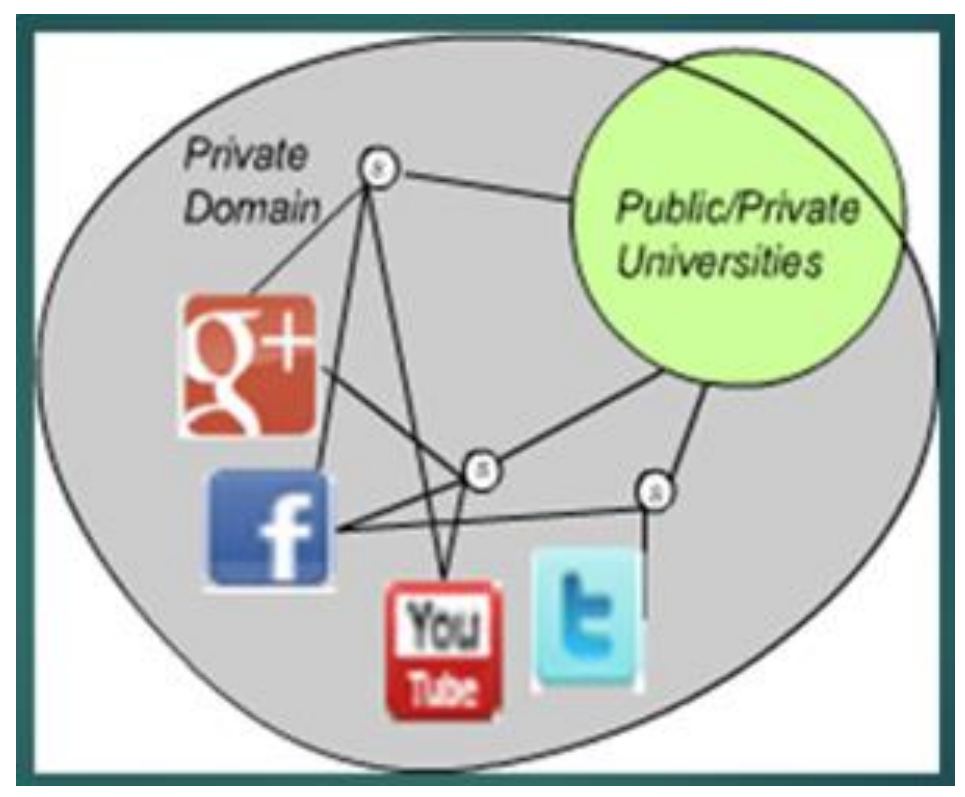

Figura 8: Ecología social de redes y universidades

\section{Los profesores}

En el mundo universitario digital se supone que los profesores universitarios se conviertan en facilitadores, expertos en medios, actores frente a una cámara, usuarios informados de las herramientas web 2.0; y además deban mantenerse al día con su propio campo académico de enseñanza. Rumble escribe: "es probable que ocurra una división del trabajo, porque a largo plazo, cualquier sistema que limite el control de diseño y entrega a una sola persona, limita tanto el alcance y sofisticación de los materiales que se pueden desarrollar, como el número de estudiantes que pueden ser apoyados. Por lo tanto, es inherentemente

El impacto económico y las posturas de los actores principales en un ámbito universitario digitalizado. Wolfram Laaser

Página 13 de 20 
ineficiente en términos de costos, dadas las economías de escala mucho mayores, en sistemas diseñados para la división del trabajo "(Rumble, 2001, p.)

Es obvio que los profesores tendrán dificultades para seguir todas estas demandas. Por lo tanto, este problema conducirá a dividir el papel del profesor en diferentes tareas que deberían ser llevadas a cabo por distintos especialistas. Por ejemplo, algunas de las conferencias en la Academia de Khan se registran con oradores profesionales o actores, en lugar de la voz del profesor. En la mayoría de los casos, las conferencias grabadas no tendrán la dinámica de las conferencias en vivo, porque los profesores serán conscientes del hecho de que el material será multiplicado y distribuido a un mayor número de usuarios; como consecuencia, algunos profesores se opondrán a tener que asumir un papel reducido en su autonomía tradicional. Pero aquellos que acepten el cambio, asumirán una tarea especializada y entrarán en un sistema coordinado de producción profesional de contenido docente; siguiendo así los caminos de la división del trabajo en la educación a distancia. Una cierta dificultad asociada al papel de ser sólo un facilitador, reside en una tendencia a presentar o moderar las opiniones de otros autores, y no a desarrollar su propia opinión. El dicho sobre "no reinventar la rueda" no es del todo válido, porque en el proceso de reinventar, el conocimiento incorporado en la invención, es reconstruido por el profesor y así mejor entendido por sí mismo.

Otro tema de importancia es la confianza que los estudiantes tendrán con sus profesores. En un tiempo en el que muchas de las funciones tradicionales de un docente universitario son cubiertas por contenido publicado en la web, fácilmente accesible para cualquiera, el educador tendrá que luchar para establecer la credibilidad. Los estudiantes parecen confiar más a menudo en sus compañeros de estudios que en sus maestros; e incluso prefieren confiar en los motores de búsqueda (World Economic Forum, 2016). Por lo tanto, al menos algunos contactos presenciales son muy importantes para crear una buena relación emocional entre los estudiantes y el docente.

\section{Los encargados de las políticas educativas}

Los responsables de la política educativa suelen estar interesados en limitar los gastos educativos sin perder eficiencia. En un entorno universitario digital tendrán la nueva opción de utilizar los recursos denominados "big data", para influir y gestionar sobre todo el sector. Anteriormente, los principales elementos de información eran los datos de matrícula, el número de graduados y programas, los salarios de los empleados, y el número de publicaciones de investigación. Hoy en día, la gran cantidad de datos digitales que se ponen a disposición al comprobar en detalle todos los procesos en el flujo de trabajo, ofrecen amplias posibilidades para utilizarlos como insumos en la administración del sector o la institución.

Un requisito importante es que los bancos de datos sean compatibles y que las aplicaciones para administrar la información puedan ser empleadas de manera correcta. Para ser efectivos, la universidad digitalizada necesita redefinir los procesos informáticos; lo que a menudo implica ajustes estructurales hacia la descentralización de organizaciones normalmente jerárquicas, respecto a los flujos de trabajo, tanto académicos como administrativos. De lo contrario, no podrá reaccionar rápida y flexiblemente al entorno cambiante (Laaser, 2011). En tal sentido, recientemente se realizaron entrevistas con personal directivo de cuatro universidades europeas respecto a las mencionadas

El impacto económico y las posturas de los actores principales en un ámbito universitario digitalizado. Wolfram Laaser

Página 14 de 20 
innovaciones, pero los resultados parecen ser un tanto contradictorios, con ausencia de estrategias claras (Bell, Douce, Caeiro, Teixeira, Martín-Aranda y Otto, 2017).

\section{Globalización de la educación superior}

¿Quién está interesado en la educación digital? ¿Es el alumno, el personal docente, el consumidor, o la industria? Como se ha señalado, casi no hay disciplina académica que esté tan estrechamente relacionada con la industria, como lo es la tecnología educativa. La mayoría de las conferencias académicas sobre dicha temática están patrocinadas y acompañadas por una exposición de vendedores de software y hardware; y las presentaciones frecuentemente siguen un paradigma simple de modernización. El interés principal parece ser el descubrir cómo las aplicaciones educativas pueden ser reconocidas y aplicadas en los nuevos productos desarrollados para el mercado de consumo. El resultado suele ser que aspectos críticos como la detección de ideologías ocultas o ciertos problemas éticos incluidos en las estrategias de los proveedores, no son muy frecuentes.

Hoy observamos que el mercado global educativo es el blanco de los grandes proveedores de tecnología; la traducción en línea es cada vez más rápida y fácil de acceder, y la localización geográfica se ha vuelto muy fácil. Mientras que muchas ideologías subyacentes a menudo se emplean para describir por ejemplo, cuestiones de globalización mediante expresiones como "aldea global".

En otro sentido, la noción de que el individuo puede controlar mejor su progreso de aprendizaje, implica que la autoridad representada por el profesor se considera un obstáculo en lugar de ser un socio o facilitador. La misma ideología subyace en la afirmación de que el grupo de pares es el mejor maestro. El Estado y sus instituciones académicas tienen que volver a una política que represente las necesidades sociales de toda la población; no necesariamente coincidentes con el interés y los objetivos de las industrias. Los MOOCs fueron concebidos como un medio de fácil acceso para todos, lo que significa socializar un bien que antes era sólo accesible a unos pocos.

También es cierto que el conocimiento de las universidades estadounidenses se abrió un poco y fue exportado internacionalmente a un precio subsidiado. Por tal motivo, probablemente perderá parte de su rigor académico asemejando el aprendizaje al "edutainment", acompañado de anuncios publicitarios (Bradford \& Loble, 2016).

En otro sentido, los XMOOC han sido criticados por la Unión Europea debido a su bajo apoyo al contenido de la enseñanza, impartido principalmente a través de conferencias grabadas (EADTU, 2015). Sin embargo, en Europa las diferencias nacionales son sustanciales, y sin financiación especial, los MOOC no sobrevivirán en los EE.UU. ni tampoco en Europa.

\section{Conclusiones}

En este artículo hemos intentado demostrar que el futuro de una "universidad digital" es una tendencia gradual hacia un aprendizaje más autónomo, individualizado, interactivo y rico, en términos de comunicación; que se remonta a los inicios de la educación a distancia, después de la Segunda Guerra Mundial. Considerar esto una revolución o un cambio disruptivo en la forma en que aprendemos y enseñamos, no puede ser confirmado. Por lo tanto, no es de extrañar que después de una discusión intensiva sobre los XMOOCs, la conclusión sigue siendo que de ninguna manera representan un enfoque disruptivo. Y

El impacto económico y las posturas de los actores principales en un ámbito universitario digitalizado. Wolfram Laaser

Página 15 de 20 
terminará como otro capítulo de idas y vueltas de la tecnología educativa, al menos en lo que se refiere a Estados Unidos.

Como afirma Audray Watters: "Después de todo, para muchas personas Edtech es un negocio, que está muy entusiasmado por la expectativa de que quizás gracias a las tecnologías, la educación en sí se está viendo cada vez más como un producto privado y de consumo, en lugar de un bien público. Creo que debemos repensar las prácticas, los sistemas de educación, y los desafíos que tenemos para Edtech, como el corporativismo y la privatización. La tecnología educativa es, después de todo, una serie de prácticas en sí; no sólo se trata de hardware o software. Edtech implica ideologías e ideas" (Watters, 2017, párrafo 7).

Rifkin escribe en su libro "The Zero Marginal Cost Society", que a diferencia de los estudiantes de Stanford, quienes tienen que pagar una cuota de unos 50.000 u\$s por año, la Udacity Online University tenía como objetivo ofrecer educación universitaria gratuita para todos, especialmente para los pobres de países en desarrollo "(Rifkin, 2014, p.171). Que los cursos sin apoyo y sin localización representen una solución a los problemas educativos de los países en desarrollo, aunque se ofrezcan de manera gratuita, es en el mejor de los casos ingenuo y nos recuerda la iniciativa "una laptop por niño" de Negroponte. Mientras tanto, los cursos de Udacity y otros MOOC americanos ya no son gratuitos.

La discusión de las posibles influencias ha demostrado que el futuro continúa lleno de ideas conflictivas y contradictorias. Un simple paradigma de modernización según el cual el sistema educativo sólo tiene que invertir en la última tecnología sería engañoso. La historia está dirigida por los intereses de las partes interesadas, y por su capacidad de resolución de conflictos y habilidad de cooperación; las consideraciones económicas tienen una influencia crucial en este proceso.

La red educativa tecnológica se basa en la idea de una economía libre, considerando a la educación como un bien comercializable. Por lo tanto, supone que los gobiernos privatizaran el sector educativo, aunque hasta ahora la educación ha sido considerada como un bien público, y no debe ser determinada por grupos de intereses especiales. Y dado que las características del software educativo tienen cierta tendencia hacia los monopolios naturales, podemos esperar un mercado educativo global controlado por unos pocos actores. Si esto se vuelve realidad en el futuro, dependerá en gran parte de lo que hagan las universidades, docentes y alumnos luchando por sus intereses. Cuál será el resultado de estas luchas por el futuro de la "universidad digital", difícilmente puede adivinarse mirando una bola de cristal.

\section{Agradecimientos}

El autor agradece a F. Schaffer por una enriquecedora discusión respecto a la parte económica y a E. A. Toloza por sus comentarios útiles y aporte en la edición del trabajo.

\section{Declaración}

El autor no informó ningún potencial conflicto de intereses.

El impacto económico y las posturas de los actores principales en un ámbito universitario digitalizado. Wolfram Laaser

Página 16 de 20 
Presentación del artículo: 2 de diciembre de 2017

Fecha de aprobación: 15 de marzo de 2018

Fecha de publicación: 31 de marzo de 2018

Laaser, W. (2018). El impacto económico y las posturas de los actores principales en un ámbito universitario digitalizado. RED. Revista de Educación a Distancia, 57(3). Consultado el (dd/mm/aaaa) en http://www.um.es/ead/red/57/laaser.pdf

\section{Financiación}

Esta investigación no ha recibido ninguna subvención específica de los organismos de financiación en los sectores públicos, comerciales o sin fines de lucro.

\section{Referencias bibliográficas}

Allen I. E. \& Seaman J. (2013). Changing Course. Ten Years of Tracking Online Education in the United States. Babson Survey Research Group and Quahog Research Group, LLC

Baggaley, J. (2013). When prophecy fails. Distance Education Vol. 34, No. 1, p. 119-128

Baggaley, J. (2016). Sandcastle competitions. Distance Education Vol. 37, No. 3 366-375)

Bates, A. (2014, July 27). Why lectures are dead (or soon will be). Blogpost: Online Learning and Distance Education Resources. Retrieved from https://www.tonybates.ca/2014/07/27/why-lectures-are-dead-or-soon-will-be/

Bell, C., Douce C. , Caeiro, S., Teixeira, A. Martín-Aranda, R. \& Otto,D. (2017). Sustainability and distance learning: a diverse European experience? pages: 1-8. DOI: $10.1080 / 02680513.2017 .1319638$

Bogost, I. (2013). The condensed classroom [Blog Post]. The Atlantic. Retrieved from http://www.theatlantic.com/technology/archive/2013/08/the-condensedclassroom/279013/

Brabazon, T. (2007). The University of Google: Education in the post-information age. Aldershot.

Bradford, K. \& Loble, M. (2016). M. Classroom Hollywood: Using popular Entertainment to Engage New MOOC Audiences. MOOCs European Stakeholder Summit 2016. 365-373

El impacto económico y las posturas de los actores principales en un ámbito universitario digitalizado. Wolfram Laaser

Página 17 de 20 
Cooch, M., Foster, H. \& Costello, E. (2015). Our Moocs with Moodle. Position paper for European cooperation on MOOCs. EADTU 2015

Creelman, A. (2014). The corridor of uncertainty. Norwegian Mooc Commission. Retrieved from http://acreelman.blogspot.de/2014/06/norwegian-mooccommission.html

Daniel, J. (2012). Making sense of MOOCs: Musings in a maze of myth, paradox, and possibility. Journal of Interactive Media in Education. Retrieved from http://jime.open. ac.uk/article/view/259

EADTU (2015). Position Paper for European Cooperation on Moocs. Jansen \& Texeira (Eds.), March 2015, Brussels

Endlich, A. \& Sohoni, V. (2017). Burned by the Bots: Why Automation is Stumbling. Digital Mc Kinsey, May 24, 2017

Gartner (2016). Gartner's 2016 Hype Cycle for Emerging Technologies Identifies Three Key Trends that Organizations Must Track to Gain Competitive Advantage. Newsroom, August 16, $2016 \quad$ Retrieved from http://www.gartner.com/newsroom/id/3412017

Hart, J. (2015). Learning in the Social Enterprise. Retrieved from http://c4lpt.co.uk/resources/litw/

Hassan, R. (2004). Media, Politics and the Network Society. McGraw-Hill Education. UK

Jansen, D. \& Schuwer, R. (2014). (eds). Institutional MOOC strategies in Europe. Status report based on a mapping survey conducted in October - December 2014. EADTU, February 2015

Jansen, D. \& Goes-Daniels (2016). Comparing Institutional MOOOC Strategies. EADTU 2015

Jones, B. (2017) eLearning Trends of 2017. eLearning Trends Art 23.2.2017

Kaltura (2018), Statement by Rubenstein J , Kaltura Headquarters

Laaser, W. (2008). Economics of Distance Education Reconsidered. The Turkish Online Journal of Distance Education (TODJE), Vol. 9, No. 3. July 2008

Laaser, W. (2011) De la unidad didáctica a la web colaborativa. La evolución de la apropiación de los medios en el contexto de la educación superior $V E s C$ (Virtualidad, Educación y Ciencias, No. 3, 2011

El impacto económico y las posturas de los actores principales en un ámbito universitario digitalizado. Wolfram Laaser

Página 18 de 20 
Laaser W. (2014). The rise and fall of the "Massively Open Online Courses" (Review article). SEEJPH 2014, posted: 11 November 2014. DOI 10.12908/SEEJPH-201433

Laaser, W. \& E. A. Toloza (2017) The Changing Role of Educational Video in Higher Distance Education, IRRODL Vol. 18, N.2 April 2017

Laaser, W (2011). Some structural changes on the way towards eUniversity. Turkish Online Journal of Distance Education, Jan. 2011, Vol. 12, No. 1

Larivière, V. , Haustein, S. \& Mongeon P. (2015). The Oligopoly of Academic Publishers in the Digital Era. PloS One, 10 (6); DOI: 10.1371

Li, F. \& Chen, X. (2012). Economies of Scope in Distance Education. IRRODL Vol. 13,3

Morgan, J. (2013). Universities challenged: The impact of Digital Technology on Teaching and Learning. Educational Innovation Position Paper, Universitas 21, 2013

NMC (2017). Horizon Report 2017 Higher Education Edition. Retrieved from https://www.nmc.org/publication/nmc-horizon-report-2017-higher-educationedition/

OEB News (2017). How do we make education a "practice of freedom"? - talking to Audrey Watters. OEB News

Rifkin J. (2016). Die Null Grenzkosten Gesellschaft. Fischer Verlag, Frankfurt

Rumble G. (2001). The Costs and Costing of Networked Learning. JALN Vol. 5 (2)

Shacklock, X. (2016). From Bricks to Clicks. The Potential of data analytics in higher education. Higher Education Commission, London

Sharples, M., de Roock, R., Ferguson, R., Gaved, M., Herodotou, C., Koh, E., KukulskaHulme, A., Looi, C-K, McAndrew, P., Rienties, B., Weller, M., Wong, L. H. (2016). Innovating Pedagogy 2016: Milton Keynes: The Open University

Shah, D. (2017) A Proposal to Put the 'M' Back in MOOCs, EdSurge News Oct. 24, 2017

Shy, O. (2001). The economics of network Industries. Cambridge University Press

Siemens, G. Gaševic \& Dawson, S. (2015). Preparing for the digital university: A review of the history and current state of distance, blended and online learning. Retrieved from http://linkresearchlab.org/preparingdigitaluniversity.pdf

Wagner, L. (1972). The Economics of the Open University. Higher Education 1. 2:159-183

El impacto económico y las posturas de los actores principales en un ámbito universitario digitalizado. Wolfram Laaser 
Watters, A. (2017). What's on the Horizon (Still, again, always) for Ed-Tech. Retrieved from http://hackeducation.com/2017/02/16/horizon

Williams, K., Kear, K., \& Rosewell, J. (2012). Quality Assessment for E-learning: a Benchmarking Approach (2nd ed.). Heerlen, The Netherlands: European Association of Distance Teaching Universities (EADTU).

World Economic Forum (2016). Digital Media and Society: Implications in a Hyperconnected Era. Report, Geneva, Switzerland

Yerbamrung, P. (1994), The emerging electronic university: A study of student cost effectiveness Economics of Distance Education, Dharnarajan et al. (Eds.), Hongkong

Young, J.R. (2017). Udacity Official Declares MOOCs 'Dead'(Though the company still offers them). EdSurge News Oct. 12, 2017

\section{Reconocimientos}

El autor agradece a F. Schaffer por una enriquecedora discusión respecto a la parte económica y a E. A. Toloza por sus comentarios útiles y aporte en la edición del trabajo.

\section{Declaración}

El autor no informó ningún potencial conflicto de intereses.

\section{Notas sobre el autor}

El Dr. Wolfram Laaser fue Director Académico en el Centro de Educación a Distancia de FernUniversität en Hagen, Alemania. Es consultor y conferencista a nivel internacional.

El impacto económico y las posturas de los actores principales en un ámbito universitario digitalizado. Wolfram Laaser 